\title{
The Stryker Regenkit PRP Kit Does Not Concentrate Canine Platelets
}

\author{
Barry Cherno ${ }^{1 *}$, Leilani Alvarez ${ }^{1}$, Kenneth E. Lamb ${ }^{2}$ and Sherman Canapp ${ }^{3}$ \\ ${ }^{1}$ Integrative and Rehabilitative Medicine Department, Animal Medical Center, New York, NY, United States, ${ }^{2}$ Lamb Statistical \\ Consulting LLC, West Saint Paul, MN, United States, ${ }^{3}$ Veterinary Orthopedic and Sports Medicine, Annapolis Junction, MD, \\ United States
}

\section{OPEN ACCESS}

Edited by:

Thomas Gadegaard Koch, University of Guelph, Canada

Reviewed by:

Maria Fahie,

Western University of Health Sciences, United States

Lauren Virginia Schnabel, North Carolina State University, United States

*Correspondence: Barry Cherno barry.cherno@amcny.org

Specialty section:

This article was submitted to Veterinary Regenerative Medicine,

a section of the journal

Frontiers in Veterinary Science

Received: 26 April 2020

Accepted: 21 September 2020

Published: 22 October 2020

Citation:

Cherno B, Alvarez L, Lamb KE and Canapp S (2020) The Stryker Regenkit PRP Kit Does Not Concentrate Canine

Platelets. Front. Vet. Sci. 7:555909.

doi: 10.3389/fvets.2020.555909
Platelet Rich Plasma (PRP) works to recruit growth factors, anti-inflammatory mediators, and blood supply to an area that may not heal well under normal conditions (e.g., joints, tendons, ligaments). Previous research has demonstrated that various PRP systems create vastly different final products. The objective of this study was to evaluate the cellular composition of the final plasma product using the Stryker RegenKit system in dogs. A peripheral blood sample was obtained from ten healthy adult dogs and compared to the final plasma product. A standard CBC was performed prior to testing and an additional sample was processed according the manufacturing guidelines for obtaining PRP ( $10 \mathrm{ml}$ total blood). Comparisons of mean platelet count, erythrocyte count, and leukocyte count were made between the peripheral blood sample and the final plasma product. Results showed that there was no significant difference in platelet count between the peripheral blood CBC and final PRP CBC ( $p=0.349$ ). There were significantly fewer erythrocytes and leukocytes in the final plasma product when compared with peripheral blood $(p=<0.0001, p=0.0318$, respectively). These results indicate that the Stryker Regenkit PRP Kit decreases leukocyte and erythrocyte concentrations but does not consistently change platelet concentrations in its final plasma product. This study, in combination with results from similar studies, may allow clinicians to better choose the appropriate PRP system to treat various musculoskeletal conditions in dogs.

Keywords: platelet rich plasma (PRP), Stryker, Regenkit, autologous, PRP

\section{INTRODUCTION}

There has been a growing interest by both pet owners and clinicians in regenerative medicine, specifically platelet rich plasma. Platelet Rich Plasma (PRP) is becoming increasingly common in canine medicine for the treatment of soft tissue injury, wound healing, bone healing, tendon and ligament injury, and for patients experiencing clinical symptoms of osteoarthritis (1-9). The benefit of PRP administration is attributed to the high concentration of growth factors contained within the alpha granules released when platelets are activated. These alpha granules release a variety of growth factors, including but not limited to, platelet-derived growth factor, insulin-like growth factor, transforming growth factor- $\beta 1$ and $\beta 2$, vascular endothelial growth factor, basic fibroblastic growth factor, and epidermal growth factor (10-12).

It is generally accepted that there are four classifications of Platelet Rich Plasma (13). These can be described as: Pure PRP or leukocyte-poor platelet rich plasma (P-PRP), Leukocyte- and Platelet-rich Plasma (L-PRP), Pure Platelet-Rich Fibrin or Leukocyte-Poor 
Platelet-Rich Fibrin (P-PRF), and Leukocyte- and PlateletRich Fibrin (L-PRF). Pure PRP, which was originally used for transfusions, typically contains $0.5 \times 10^{11}$ platelets per unit (13). These can be injected (intra-articular) or used topically (as a gel for wounds). These concentrates are most commonly produced using continuous flow plasmapheresis and are devoid of leukocytes and have a low-density fibrin network. Leukocyteand Platelet-rich Plasma (L-PRP) also have a low-density fibrin network; however, unlike Pure PRP, this concentration contains leukocytes. This type of PRP can be either injected or used topically. Pure Platelet-Rich Fibrin (P-PRF) has a high-density fibrin network and no leukocytes. These preparations are more of a solid consistency and therefore are used topically as a gel and cannot be injected. Finally, Leukocyte- and Platelet-Rich Fibrin (L-PRF) is another high-density fibrin network. Unlike P-PRF, these concentrates do contain leukocytes. Similarly, to P-PRF, due to the high-density fibrin network, these preparations are more solid and used topically and are not capable of being injected (14). Some will further categorize PRP into leukocyte-rich PRP (LR-PRP) vs. leukocyte-poor PRP (LP-PRP). LR-PRP is defined as having a neutrophil concentration above baseline while LPPRP has a neutrophil concentration below baseline (15). Mishra et al. (16) went a step further in classifying PRP products, not only by the presence of leukocytes, but by separating those that contain $>5$ times the platelet concentration compared to whole blood (Type A) from those than contain $<5$ times the platelet concentration compared to whole blood (Type B).

Many systems exist to process a PRP product in human and equine medicine fields as well as multiple canine specific systems. These systems have been shown to produce products differing significantly in platelet, red blood cell (RBC), and white blood cell (WBC) concentrations (12, 17-19). Each commercially available PRP system comes with its own set of instructions regarding preparation. One of the most important aspects of producing a PRP product is centrifugation. This process is what separates the different cell types (erythrocytes, leukocytes, and platelets) by gravitational force $(G)$ into layers based on density so that they can be preferentially selected for what the manufacturer suspects to be the most ideal PRP concentrate. The $\mathrm{G}$ is determined by the revolutions per minute (RPM) and radius of the rotor. The time and RPM each tube of blood and anticoagulant is subject to is different between the systems. Some systems use a single "soft spin" (slower rotations, lower G) or single "hard spin" (faster rotations, higher $\mathrm{G}$ ), while others undergo a combination or double spin (often a soft spin followed by removal of the erythrocyte layer and then a hard spin). Studies have shown that the single spin method packs erythrocytes to the bottom of the test tube to be discarded. A "hard spin" will also begin to separate leukocytes from platelets. The double spin allows for the platelets to be separated further from the plasma in the form of pellets for improved recovery $(20,21)$.

The make-up of an ideal PRP product (concentration of platelets, neutrophils, macrophages, total leukocytes, and erythrocytes) remains unknown. The purpose of this study was not to determine the makeup of an ideal PRP product, but rather to evaluate the cellular composition of the final product of the Stryker Regenkit PRP kit, specifically in regard to the platelet,
$\mathrm{RBC}$, and WBC concentrations. As future studies continue to gather evidence of what PRP product components are best for varying conditions, information garnered from the study reported here, can be used to choose which system would be best for particular syndromes.

\section{MATERIALS AND METHODS}

Ten adult healthy dogs were prospectively recruited for the study. Informed owner consent was obtained from all participating subjects and the study protocol was approved by the Animal Medical Center Institutional Animal Care and Use Committee. Dogs were considered for inclusion if no abnormalities were identified on physical examination by the primary investigator the same day of the study or abnormalities in CBC obtained within a month of the study. All subjects were $>15 \mathrm{~kg}$ in weight, neutered or intact with age range $>1 \mathrm{yr}<12$ years. Normal pre-screening $\mathrm{CBC}$ was confirmed on the same day as study collection. Any subject who had significant alterations in CBC or that required sedation to obtain the necessary blood sample was excluded.

Once results of the pre-screening blood work returned with no abnormalities, adequate samples of blood $(10 \mathrm{ml}$ peripheral whole blood from the jugular vein) were obtained by one of two certified veterinary technicians. A sample of $2 \mathrm{ml}$ of whole blood was submitted for a comprehensive CBC (Idexx ${ }^{\circledR}$ 2 test code 326) and $8 \mathrm{ml}$ was used as per Stryker PRP Kit manufacturer guidelines as follows. The same investigator (BC) processed all the samples as described here per manufacturer instructions. The $8 \mathrm{ml}$ of whole blood was placed in the RegenTHT tube and inverted gently 4-5 times to mix the anticoagulant additive with the blood. The RegenTHT tube was then inserted into the centrifuge with a measured counterbalance tube filled with an equal amount of water. The lid was closed and set for 1,500 Relative Centrifugal Force (3400 RPM) for $8 \mathrm{~min}$. When the centrifugation was completed, the RegenTHT tube was removed and inverted 5 times to resuspend the platelets in the plasma supernatant. The supernatant fraction (final plasma product) was then collected with an 18 gauge needle on a $6 \mathrm{ml}$ syringe. A final comprehensive $\mathrm{CBC}$ was then performed immediately on the final product from the Stryker kit. Comparisons in platelet, WBC, and RBC counts were made between the peripheral CBC (jugular blood collected at same time as the PRP sample) and the final Stryker plasma product for each dog.

\section{Statistical Methods}

Baseline descriptive statistics are reported as mean and standard deviation. The distribution of residuals was assessed by visual inspection followed by Kolmogorov Smirnoff test. Between-groups and within group analyses were performed using independent and dependent model analysis of variance (ANOVA) where applicable as error residuals were normally distributed. All analyses were carried out using SAS 9.4 statistical software (Cary, NC) where $p<0.05$ was deemed significant. 
TABLE 1 | Mean and standard deviation cell counts in CBC of peripheral blood compared with CBC of final processed PRP product in 10 healthy canines using Stryker Regenkit PRP Kit.

\begin{tabular}{lccr}
\hline & $\begin{array}{c}\text { Peripheral } \\
\text { blood CBC }\end{array}$ & $\begin{array}{c}\text { Final PRP } \\
\text { product CBC }\end{array}$ & p-value \\
\hline Platelets (K/uL) & $258.8 \pm 73.2$ & $179.9 \pm 249.0$ & 0.349 \\
Lymphocytes (/uL) & $2765.7 \pm 1141.8$ & $2575 \pm 1990.8$ & 0.7957 \\
Monocytes (/uL) & $372.1 \pm 157.7$ & $279.9 \pm 367.9$ & 0.4758 \\
Neutrophils (/uL) & $5150.3 \pm 1593.2$ & $2303.9 \pm 1335.0$ & 0.0004 \\
Red Blood Cells (M/UL) & $7.2 \pm 0.4$ & $0.05 \pm 0.04$ & $<0.0001$ \\
White Blood Cells (K/uL) & $8.6 \pm 1.9$ & $5.7 \pm 3.5$ & 0.0318 \\
\hline
\end{tabular}

TABLE 2 | Platelet counts in CBC of peripheral blood compared with platelet counts in final processed PRP product in healthy canines using Stryker Regenkit PRP Kit.

\begin{tabular}{lcc}
\hline Patient & $\begin{array}{c}\text { Peripheral blood } \\
\text { Platelet count (K/uL) }\end{array}$ & $\begin{array}{c}\text { Processed PRP } \\
\text { Platelet count (K/uL) }\end{array}$ \\
\hline 1 & 226 & 234 \\
2 & 320 & 118 \\
3 & 404 & 854 \\
4 & 254 & 103 \\
5 & 215 & 221 \\
6 & 130 & 88 \\
7 & 256 & 12 \\
8 & 294 & 122 \\
9 & 277 & 15 \\
10 & 212 & 32 \\
\hline
\end{tabular}

\section{RESULTS}

There was no statistically significant difference between concentrations of platelets for screening $\mathrm{CBC}$ on peripheral blood and the final plasma product $(p=0.3490)$. The mean platelet concentration on the peripheral blood samples was $258.8 \mathrm{~K} / \mathrm{uL}$ while the mean concentration of platelets in the final product was $179.9 \mathrm{~K} / \mathrm{uL}$ (Table 1). Furthermore, the results showed that not all final plasma products had higher platelet concentrations compared to the screening $\mathrm{CBC}$ for the same subject. Seven of the ten samples had lower concentrations of platelets in the final product, while only three had increased platelet concentrations in the final plasma product (Table 2).

There were significantly less white blood cells found in the processed plasma product compared to screening CBC peripheral blood sample $(p=0.0318$; Table 1). There were significantly less neutrophils found in the processed sample compared to the peripheral blood ( $p=0.0004$; Table 1). There was no statistically significant difference in monocytes or lymphocytes between the peripheral blood and processed plasma product ( $p=0.4758$ and 0.7957 , respectively; Table 1 ).

The greatest difference between samples was found in red blood cell count. The mean concentration of red blood cells was significantly less in the final plasma product $(0.045 \mathrm{M} / \mathrm{uL})$
TABLE 3 | Subject demographics of adult healthy dogs used to test PRP product using Stryker Regenkit.

\begin{tabular}{lccc}
\hline Patient & Age (years) & Sex & Weight (kg) \\
\hline 1 & 4.5 & M & 19.70 \\
2 & 3 & FS & 23.10 \\
3 & 9 & FS & 27.20 \\
4 & 3 & NM & 19.80 \\
5 & 2 & NM & 34.00 \\
6 & 3 & NM & 24.70 \\
7 & 10 & NM & 17.70 \\
8 & 7 & NM & 35.50 \\
9 & 4 & NM & 18.70 \\
10 & 3 & FS & 17.60 \\
\hline
\end{tabular}

TABLE 4 | Platelet:Leukocyte and Platelet:Neutrophil Ratios in the Final PRP Product of each dog and Mean \pm Standard Deviation of ratios Using the Stryker Regenkit PRP Kit.

\begin{tabular}{lcc}
\hline Dog & Platelet:Leukocyte ratio & Platelet:Neutrophil ratio \\
\hline 1 & 41.05 & 0.15 \\
2 & 62.11 & 0.1 \\
3 & 108.1 & 0.21 \\
4 & 28.61 & 0.06 \\
5 & 17.97 & 0.04 \\
6 & 22 & 0.04 \\
7 & 1.29 & 0.005 \\
8 & 174.29 & 0.27 \\
9 & 2.54 & 0.005 \\
10 & 6.04 & 0.02 \\
Mean \pm SD & \pm 55.52 & $0.091 \pm 0.092$ \\
\hline
\end{tabular}

compared to peripheral blood $(7.192 \mathrm{M} / \mathrm{uL} ; p=<0.0001$; Table 1).

Despite there being two times as many males than females in the study population, the frequency of males (12) vs. females (22) was not found to be significantly different ( $p=0.2059$; Table 3 ).

The platelet:leukocyte and platelet:neutrophil ratios in the final plasma product were also evaluated between dogs (Table 4). The platelet:leukocyte ratio ranged from 2.54 to 108.1 with a mean of 46.4 and standard deviation of 55.5. The platelet:neutrophil ratio ranged from 0.005 to 0.27 with a mean of 0.091 and standard deviation of 0.092 .

\section{DISCUSSION}

In the present study, there was no significant difference in concentration of platelets when peripheral blood was compared to the final plasma product of the Stryker system. Previous literature suggests that a 3-5-fold increase in platelet concentration is desirable for an optimal PRP product (23). In the study performed here, the mean concentration of platelets following centrifugation was actually lower than the mean concentration of platelets in the screening CBC (Table 1). Only 
3 of the 10 subjects demonstrated a final plasma product with a higher concentration of platelets when compared to the initial $\mathrm{CBC}$ on peripheral blood (prior to processing). Unlike the system studied here, Perego et al. (24) showed that the CPUNT 20 system consistently concentrates canine platelets. However, the Stryker RegenKit PRP system is not the only system of its kind that was shown not to concentrate platelets efficiently. In a study by Carr et al. (12) two of the five systems investigated did not see significant changes in PRP platelet concentrations compared to whole blood. Similarly, Franklin et al. (18) found one of five systems evaluated did not concentrate platelets. The product tested in this study may therefore not provide the suspected beneficial effects of healing, tissue regeneration, and analgesic effects brought about by the presence of platelets in a final PRP concentrate $(10,25)$.

The current study showed that the final plasma product of the Stryker system resulted in significantly less white blood cells compared to peripheral blood. This is similar to two of five systems in one study (12) and two of five in another (18). In a study carried out by Perego et al. (24) evaluating the CPUNT 20 system, the final product did not have a significantly different leukocyte count compared with whole blood. This system; however, has two settings which gives the user the option of allowing or disallowing leukocytes in the final product. From previous studies, we know that leukocytes lead to catabolism of tissue while also promoting an inflammatory response (10, 26). In cases with infected wounds, it may be beneficial to introduce leukocytes to the site of healing (4). Increases in matrix metalloproteinase-13 also promotes degradation of tendon and ligament $(10,26)$. Braun et al. (22) showed increased pain, cell death, and synoviocyte activation in PRP products what were leukocyte rich (22). Therefore, increased leukocyte counts would likely be less ideal in a final PRP product being used for tendon, ligament, bone, and/or osteoarthritis cases $(4,10,14,26)$.

There were less neutrophils following processing in the Stryker system compared to peripheral blood; however, there was no significant difference in concentrations of monocytes or lymphocytes. To draw a comparison between the system evaluated here to those from previous studies, three of five systems examined by Carr et al. (12) and the system evaluated by Perego et al. (24) also significantly reduced neutrophil concentrations. Only two of the five systems final products examined by Carr et al. (12) had no significant change of monocyte concentration compared to whole blood (two had significantly decreased concentrations and one had an increased concentration) and all had significantly different concentrations of lymphocytes compared to whole blood (two had significantly decreased concentrations and three had an increased concentration). The CPUNT 20 system's final PRP product had an increased concentration of both monocytes and lymphocytes when compared to whole blood (24). This shows the vast variability of numbers and types of leukocytes present across the various final products produced by the different machines. It is suspected that neutrophils would be beneficial for cases involving infectious processes; however, they can also cause undesirable tissue destruction. Monocytes, on the other hand, divide into macrophages and may be desirable in a PRP product meant for musculoskeletal repair due to their ability to clear harmful debris from injured tissue and other anabolic effects (10).

The study reported here found significantly less red blood cells in the Stryker plasma product when compared to peripheral blood. This seems to be fairly consistent across many PRP systems as all systems evaluated by Carr et al. (12) and Perego et al. (24) also significantly reduced erythrocyte concentrations in the final product when compared to whole blood. Franklin et al. (18) did not report erythrocyte count, rather hematocrit and therefore was not compared to the results of this study. It remains unknown if an ideal platelet rich plasma product should be devoid of red blood cells. Previous studies report that RBCs may cause direct cartilage damage, stimulate synoviocytes to release catabolic mediators, and may be cytotoxic due to free radical induced apoptosis $(10,22)$. Despite possible benefits of their ability to bring oxygen to tissues and promote vasodilation, it is likely that they are undesirable for most cases of musculoskeletal injury.

The results of this study indicate that the final product of the Stryker RegenPRP Kit did not produce what can be defined as a PRP product from canine blood. As it was unable to consistently concentrate platelets, it does not fall into one of the four general PRP categories (P-PRP, L-PRP, P-PRF, and L$\mathrm{PRF})$. We suspect that this is due to the processing with a single 8-min "soft" spin session at 1,500 g. Had a harder spin been performed, better separation of platelets from leukocytes may have been appreciated. Had second spin been performed, better and more efficient separation of platelets may have been possible. However, additional studies would need to be performed to substantiate these assumptions. Using the classification system discussed in the introduction, the final Stryker Regenkit plasma product would be considered closest to the Leukocyte Poor PRP (LP-PRP) as all final plasma products contained lower numbers of neutrophils compared to the peripheral blood cell count following processing. This classification of product is similar to the final product produced by two of the five systems evaluated by Frankin et al. (27).

In the presented study, no consistency was found between dogs for either platelet:leukocyte ratio or platelet:neutrophil ratio. Franklin et al. (18) discusses consistency of platelet:leukocyte ratios across 5 different PRP machines; however, no studies could be found by the author that evaluates these ratios between canine samples using the same machine.

It is worth noting that no previous validation studies for canines or any other species using the Stryker Regekit PRP Kit could be found on literature search. Requests to the manufacturers requesting the data and studies were not answered. As no previous literature could be found on the Stryker Regenkit PRP system in any species, the authors were unable to compare the findings of this study with those of others performed. A major limitation of this study is the small number of dogs used. This may lead to type II errors in our data. Further studies with increased number of subjects would be ideal.

As ideal PRP product compositions are learned for differing conditions, the authors feel it is important that any company manufacturing such a system perform, and make available, a 
3rd party prospective analysis of their biologics/devices prior to advertisement and sale. It is also recommended that clinicians perform and record pre- and post-spin analysis on all PRP products that are processed to ensure the most appropriate preparation is being used and can be tracked over time.

\section{DATA AVAILABILITY STATEMENT}

The raw data supporting the conclusions of this article will be made available by the authors, without undue reservation.

\section{ETHICS STATEMENT}

The animal study was reviewed and approved by Animal Medical Center Institutional Animal Care and Use Committee.

\section{REFERENCES}

1. Abouelnasr K, Hamed M, Lashen S, El-Adl M, Eltaysh R, Tagawa M. Enhancement of abdominal wall defect repair using allogenic platelet-rich plasma with commercial polyester/cotton fabric (Damour) in a canine model. J Vet Med Sci. (2017) 79:1301-9. doi: 10.1292/jvms.17-0139

2. Canapp SO Jr, Canapp DA, Ibrahim V, Carr BJ, Cox C, Barrett JG. The use of adipose-derived progenitor cells and platelet-rich plasma combination for the treatment of supraspinatus tendinopathy in 55 dogs: a retrospective study. Front Vet Sci. (2016) 3:61. doi: 10.3389/fvets.2016. 00061

3. Canapp SO Jr, Leasure CS, Cox C, Ibrahim V, Carr BJ. Partial cranial cruciate ligament tears treated with stem cell and platelet-rich plasma combination therapy in 36 dogs: a retrospective study. Front Vet Sci. (2016) 3:112. doi: $10.3389 /$ fvets.2016.00112

4. Farghali HA, AbdElKader NA, Khattab MS, AbuBakr HO. Evaluation of subcutaneous infiltration of autologous plateletrich plasma on skin-wound healing in dogs. Biosci Rep. (2017) 37:BSR20160503. doi: 10.1042/BSR20160503

5. Franklin SP, Burke EE, Holmes SP. The effect of platelet-rich plasma on osseous healing in dogs undergoing high tibial osteotomy. PLoS ONE. (2017) 12:e177597. doi: 10.1371/journal.pone.01 77597

6. Upchurch DA, Renberg WC, Roush JK, Milliken GA, Weiss ML. Effects of administration of adipose-derived stromal vascular fraction and platelet-rich plasma to dogs with osteoarthritis of the hip joints. Am J Vet Res. (2016) 77:940-51. doi: 10.2460/ajvr.77.9.940

7. Yun S, Ku SK, Kwon YS. Adipose-derived mesenchymal stem cells and platelet-rich plasma synergistically ameliorate the surgicalinduced osteoarthritis in Beagle dogs. (2016) J Orthop Surg Res. 11:9. doi: 10.1186/s13018-016-0342-9

8. Hsu WK, Mishra A, Rodeo SR, Fu F, Terry MA, Randelli P, et al. Platelet rich plasma in orthopaedic applications: evidence-based recommendations for treatment. J Am Acad Orthop Surg. (2013) 21:739-48. doi: 10.5435/00124635-201312000-00004

9. Brossi PM, Moreira JJ, Machado T, Baccarin R. Platelet-rich plasma in orthopedic therapy: a comparative systematic review of clinical and experimental data in equine and human musculoskeletal lesions. BMC Vet Res. (2015). 11:98. doi: 10.1186/s12917-0150403-Z

10. Boswell SG, Cole BJ, Sundman EA, Karas V, Fortier LA. Plateletrich plasma: a milieu of bioactive factors. Arthroscopy. (2012) 28:42939. doi: 10.1016/j.arthro.2011.10.018

11. Carr BJ, Canapp SO Jr, Mason DR, Cox C, Hess T. Canine plateletrich plasma systems: a prospective analysis. Front Vet Sci. (2016) 2:73. doi: $10.3389 /$ fvets. 2015.00073

\section{AUTHOR CONTRIBUTIONS}

BC, LA, and SC contributed to conception and design of the study. BC organized the database. BC and LA wrote the manuscript. KL provided statistical analysis. All authors contributed to manuscript revision, read, and approved the submitted version.

\section{ACKNOWLEDGMENTS}

The authors would like to acknowledge William Tavolacci and Caitlin Clissold for their assistance in obtaining blood samples and helping process the platelet rich plasma. The Stryker Regenkits were from an extraneous supply as they were no longer being used by the investigators hospital.

12. Filardo G, Kon E, Roffi A, Di Matteo B, Merli ML, Marcacci M. Platelet rich plasma: why intra-articular? A systematic review of preclinical studies and clinical evidence on PRP for joint degeneration. Knee Surg Sports Traumatol Arthrosc. (2013) 23:2459-74. doi: 10.1007/s00167-013-2743-1

13. Dohan Ehrenfest DM, Rasmusson L, Albrektsson T. Classification of platelet concentrates: from pure platelet-rich plasma (P-PRP) to leucocyte- and platelet-rich fibrin (L-PRF). Trends Biotechnol. (2008) 27:158-67. doi: 10.1016/j.tibtech.2008.11.009

14. Dohan Ehrenfest DM, Doglioli P, de Peppo GM, Del Corso M, Charrier JB. Choukroun's platelet-rich fibrin (PRF) stimulates in vitro proliferation and differentiation of human oral bone mesenchymal stem cell in a dose-dependent way. Arch Oral Biol. (2010) 55:185-94. doi: 10.1016/j.archoralbio.2010.01.004

15. Le Adrian DK, Enweze L, DeBaun MR, Dragoo JL. Current clinical recommendations for use of platelet-rich plasma. Curr Rev Musculoskelet Med. (2018) 11:624-32. doi: 10.1007/s12178-018-9527-7

16. Mishra A, Harmon K, Woodall J, Vieira A. Sports medicine applications of platelet rich plasma. Curr Pharm Biotechnol. (2012) 13:1185-95. doi: 10.2174/138920112800624283

17. Castillo TN, Pouliot MA, Kim HJ, Dragoo JL. Comparison of growth factor and platelet concentrations from commercial platelet-rich plasma separation systems. Am J Sports Med. (2011) 39:266-71. doi: 10.1177/0363546510387517

18. Franklin SP, Garner BC, Cook JL. Characteristics of canine platelet-rich plasma prepared with five commercially available systems. Am J Vet Res. (2015) 76:822-7. doi: 10.2460/ajvr.76.9.822

19. Frye CW, Enders A, Brooks MB, Struble AM, Wakshlag JJ. Assessment of canine autologous platelet-rich plasma produced with a commercial centrifugation and platelet recovery kit. Vet Comp Orthop Traumatol. (2016) 29:14-9. doi: 10.3415/VCOT-15-03-0046

20. Dhurat R, Sukesh MS. Principles and methods of preparation of platelet-rich plasma: a review and author's perspective. $J$ Cutan Aesthet Surg. (2014) 7:189-97. doi: 10.4103/0974-2077.1 50734

21. Shin HS, Woo HM, Kang BJ. Optimisation of a double-centrifugation method for preparation of canine platelet-rich plasma. BMC Vet Res. (2017). (2017) 13:198. doi: 10.1186/s12917-017-1123-3

22. Braun HJ, Kim HJ, Chu CR, Dragoo JL. The effect of platelet-rich plasma formulations and blood products on human synoviocytes: implications for intra-articular injury and therapy. Am J Sports Med. (2014) 42:120410. doi: $10.1177 / 0363546514525593$

23. Pelletier MH, Malhotra A, Brighton T, Walsh WR, Lindeman R. Platelet function and constituents of platelet rich plasma. Int J. Sports Med. (2013) 34:74-80. doi: 10.1055/s-0032-1316319

24. Perego R, Spada E, Baggiani L, Martino PA, Proverbio D. Efficacy of a semi automated commercial closed system for autologous leukocyte- and platelet-rich plasma (l-prp) production in dogs: a 
preliminary study. Animals. (2020) 10:1342. doi: 10.3390/ani100 81342

25. Lee M, Kim JH, Kwak HH, Woo HM, Han JH, Yayon A, et al. A placebo-controlled study comparing the efficacy of intra-articular injections of hyaluronic acid and a novel hyaluronic acid-platelet-rich plasma conjugate in a canine model of osteoarthritis. J Orthop Surg Res. (2019) 14:314. doi: 10.1186/s13018-019-1352-1

26. McCarrel T, Fortier L. Temporal growth factor release from platelet-rich plasma, trehalose lyophilized platelets, and bone marrow aspirate and their effect on tendon and ligament gene expression. J Orthop Res. (2009) 27:103342. doi: 10.1002/jor.20853

27. Franklin SP, Birdwhistell KE, Strelchik A, Garner BC, Brainard BM. Influence of cellular composition and exogenous activation on growth factor and cytokine concentrations in canine platelet-rich plasmas. Front Vet Sci. (2017) 4:40. doi: 10.3389/fvets.2017.00040
Conflict of Interest: SC is a paid consultant of Companion Animal Health, who produces a PRP kit and could be construed as a competitor to Stryker. KL is employed by the company Lamb Statistical Consulting LLC.

The remaining authors declare that the research was conducted in the absence of any commercial or financial relationships that could be construed as a potential conflict of interest.

Copyright (C) 2020 Cherno, Alvarez, Lamb and Canapp. This is an open-access article distributed under the terms of the Creative Commons Attribution License (CC BY). The use, distribution or reproduction in other forums is permitted, provided the original author(s) and the copyright owner(s) are credited and that the original publication in this journal is cited, in accordance with accepted academic practice. No use, distribution or reproduction is permitted which does not comply with these terms. 